\title{
Testicular Adrenal Rest Tumours: fisiopatologia, diagnosi e trattamento
}

\author{
Dario De Alcubierre ${ }^{1} \cdot$ Riccardo Pof ${ }^{1} \cdot$ Carlotta Pozza ${ }^{1}$
}

Accettato: 6 giugno 2020 / Pubblicato online: 8 febbraio 2021

(c) The Author(s) 2021

Sommario I testicular adrenal rest tumors (TARTs) costituiscono una causa comune di disfunzione gonadica e infertilità in pazienti affetti da iperplasia surrenalica congenita (Congenital Adrenal Hyperplasia, CAH), con una prevalenza che varia dal 14 all' $86 \%$. Dal punto di vista biochimico, istologico e molecolare, i TARTs mostrano caratteristiche tipiche delle cellule surrenaliche e per questo si è ipotizzato che derivino da una proliferazione di cellule della corteccia surrenalica in sede testicolare. Studi recenti riconducono però l'origine dei TARTs a una popolazione di cellule staminali pluripotenti adrenal-like, derivanti dalla cresta urogenitale, già presenti in sede gonadica durante l'embriogenesi, che vanno incontro a differenziazione surrenalica e ad aumentata proliferazione se sottoposti a elevati livelli di ormone adrenocorticotropo (ACTH). La loro crescita può determinare un'alterazione della funzione gonadica per compressione diretta sui tubuli seminiferi e per l'influenza sull'ambiente ormonale intratesticolare per via paracrina, risultando spesso in un quadro di azoospermia di tipo ostruttivo. La diagnosi di TARTs si avvale principalmente dell'eco Color-Doppler testicolare, ma richiede una puntuale diagnosi differenziale con lesioni morfologicamente simili, quali i tumori germinali e i tumori a cellule del Leydig, in quanto la gestione terapeutica è differente. La terapia classica si basa sull'impiego di glucocorticoidi ad alte dosi, che in alcuni ca-

Proposto da Daniele Gianfrilli.

Informazioni Supplementari La versione online contiene materiale supplementare disponibile su

https://doi.org/10.1007/s40619-021-00816-5.

$\triangle$ C. Pozza

carlotta.pozza@uniroma1.it

1 Dipartimento di Medicina Sperimentale, Sezione di Fisiopatologia Medica ed Endocrinologia, Sapienza Università di Roma, Roma, Italia si possono condurre a una regressione delle dimensioni della massa. La gestione della CAH nei soggetti che presentano TARTs richiede un approccio personalizzato con screening ecografico da adottare già a partire dall'infanzia e si avvale altresì di counseling andrologico in merito alla possibilità di preservare la fertilità tramite crioconservazione.

Parole chiave Testicular adrenal rest tumors - Iperplasia surrenalica congenita Congenital adrenal hyperplasia . Steroidogenesi surrenalica

\section{Introduzione}

I testicular adrenal rest tumors (TARTs) sono lesioni tumorali benigne a localizzazione intratesticolare caratterizzate da analogia funzionale e istologica con le cellule adrenocorticali. Rappresentano una ben nota complicanza nella popolazione maschile affetta da iperplasia surrenalica congenita (congenital adrenal hyperplasia, $\mathrm{CAH}$ ), entità nosografica che include una serie di disordini autosomico-recessivi della corteccia surrenalica caratterizzati da un'alterata produzione steroidea dovuta a difetti enzimatici della steroidogenesi.

Attualmente la prevalenza globale dei TARTs nei maschi affetti da CAH è stimata essere intorno al 37\% [1]; i dati presenti in letteratura documentano la presenza dei TARTs già in età pediatrica, con una prevalenza variabile tra il 18 e il $25 \%$ nei bambini maschi affetti da $\mathrm{CAH}$ [1], numero che incrementa durante l'adolescenza (66\%) [2], inquadrando così i TARTs come una condizione ad aumentata prevalenza nell' età puberale.

La maggior parte degli studi descrive la presenza dei TARTs nelle forme classiche di CAH. Sono comunque riportati in letteratura casi isolati di TARTs in pazienti 
con mutazioni a carico degli enzimi 11-idrossilasi e 3bidrossisteroidodeidrogenasi, così come nelle forme non classiche di CAH [3].

Ne consegue che i TARTs possono manifestarsi in tutti i genotipi di $\mathrm{CAH}$, seppur con incidenza molto variabile. $\mathrm{La}$ loro prevalenza risulta inoltre essere direttamente correlata con la severità della malattia di base: dei pazienti con TART, l' $80 \%$ presenta una forma severa di CAH, e solo il $20 \%$ una forma lieve di malattia [1].

\section{Origine e sviluppo}

L'ipotesi tradizionale attribuisce la genesi degli adrenal rest tumors a una popolazione di cellule surrenaliche aberranti che migrano in sede gonadica durante lo sviluppo embriogenetico; tali precursori, oggetto di un fisiologico processo di regressione spontanea nel corso del primo anno di vita, nei pazienti con CAH proliferano in seguito all'esposizione a concentrazioni aumentate di fattori di crescita, dando origine alle note lesioni testicolari.

Ad oggi l'ipotesi più accreditata riconduce l'origine dei TARTs a una popolazione di cellule staminali pluripotenti adrenal-like, derivanti dalla cresta urogenitale, già presenti in sede gonadica durante l'embriogenesi. Tali precursori, verosimilmente cellule del Leydig fetali esprimenti recettori per ACTH, ormone luteinizzante (LH) e angiotensina II (ATII), vanno incontro a differenziazione surrenalica e ad aumentata proliferazione se sottoposti a elevati livelli di ormone adrenocorticotropo (ACTH), come accade tipicamente nei pazienti affetti da CAH [1].

Pertanto, lo sviluppo degli adrenal rest tumors richiede un'esposizione precoce e cronica a elevati livelli di ACTH, verosimilmente già in era prenatale. Questa tesi è sostenuta da numerose evidenze: innanzitutto, la più elevata prevalenza dei TARTs nel contesto di forme severe di CAH, caratterizzate da livelli circolanti di ACTH più elevati, ma anche la loro, seppur rara, incidenza in altre condizioni accomunate da un'esposizione cronica a livelli aumentati di ormone adrenocorticotropo, quali il Morbo di Addison [4], la Malattia di Cushing e la Sindrome di Nelson [5]. Al contrario, essi non si sviluppano nei casi in cui l'esposizione a elevati livelli di ACTH si verifichi a seguito di una patologia acquisita in età adulta, come ad esempio nei pazienti che subiscono interventi di surrenectomia bilaterale.

È inoltre ben documentato in letteratura come un'inadeguata compliance del paziente o una terapia con glucocorticoidi sottodosata possano contribuire allo sviluppo dei TARTs [6]; al tempo stesso, è noto che essi possono andare incontro a regressione in seguito alla soppressione dei livelli di ACTH indotta dal trattamento con glucocorticoidi a dosaggio intensificato, sottolineando ulteriormente l'ACTH-dipendenza di tali lesioni [7].
Recenti evidenze hanno messo in luce, nella patogenesi dei TARTs, un possibile ruolo giocato da parte di altre molecole quali l'ATII e LH, il cui picco puberale contribuisce all'ulteriore proliferazione di tali cellule aberranti, spiegando così l'aumentata incidenza osservata in quella fascia di età [8].

\section{Patogenesi e presentazione clinica}

Nella maggior parte dei casi la diagnosi di CAH precede il riscontro clinico di queste lesioni. Alcuni studi, tuttavia, hanno riportato come i TARTs possano essere la prima manifestazione clinica di forme di CAH non severe: tra questi, uno studio retrospettivo condotto da Rutgers e collaboratori [9] ha analizzato 40 pazienti con TARTs, portando alla luce nel $18 \%$ dei casi un quadro di CAH non precedentemente diagnosticata.

I TARTs sono tumori benigni, bilaterali nel $77 \%$ dei casi e, specialmente nelle fasi precoci del loro sviluppo, del tutto asintomatici e non palpabili all'esame obiettivo testicolare se di piccole dimensioni, data la loro caratteristica localizzazione a livello della rete testis. Il loro principale significato clinico è sicuramente legato all'impatto che hanno sulla funzione gonadica: essi rappresentano, infatti, la principale causa di infertilità nei pazienti maschi affetti da $\mathrm{CAH}$, determinando una compromissione della spermatogenesi [10]. A tal proposito, Bouvatier e colleghi [11] hanno riportato una prevalenza significativamente maggiore di azoospermia in pazienti con TARTs rispetto a quelli che ne sono privi, mentre Falhammar et al. [12] hanno evidenziato come i pazienti con TARTs abbiano una minore probabilità di concepire figli biologici.

Numerosi studi hanno dimostrato un notevole peggioramento dei parametri seminali nei pazienti con TARTs, che tipicamente presentano un volume seminale, una concentrazione spermatica e una motilità progressiva significativamente più bassi rispetto a pazienti che non presentano tali lesioni testicolari [13].

L'impatto sulla fertilità è mediato da multipli meccanismi patogenetici: in primo luogo, la progressiva crescita dei TARTs determina, come descritto nel "modello a 5 stadi" di Claahsen e collaboratori [14], una progressiva ostruzione dei tubuli seminiferi, a lungo andare complicata dall'instaurarsi di un'infiltrazione linfocitaria e da una fibrosi peritubulare risultando, infine, in un danno testicolare irreversibile, con azoospermia ostruttiva e infertilità. Peraltro, è degno di nota che lesioni di lungo corso, a causa della prolungata compressione del parenchima testicolare sano, possano determinare intensa algia testicolare [15].

Oltretutto, i TARTs producono androgeni: un recente studio ha dimostrato la presenza di concentrazioni più 
elevate di steroidi surrenalici (androstenedione, 17-OHprogesterone, 21-deossicortisolo) nel sangue venoso spermatico rispetto al sangue venoso periferico [16]. Tale risultato ha trovato effettivamente conferma in recenti esperimenti in vitro, che hanno dimostrato la produzione di steroidi surrenalici da parte delle cellule TART [17]. Tale iperproduzione di androgeni surrenalici interferisce con la funzione testicolare sia a livello locale con effetto paracrino, sia a livello centrale, dal momento che gli elevati livelli di androgeni surrenalici (aromatizzati a estrogeni) esercitano un feedback negativo a livello ipotalamo-ipofisario, risultando nella soppressione della secrezione delle gonadotropine e determinando, quindi, un quadro di ipogonadismo ipogonadotropo [13].

Infine, la presenza dei TARTs si associa a un peggior controllo ormonale nei pazienti con CAH, con livelli di ACTH [18], 17-OH-progesterone e androstenedione significativamente più elevati rispetto ai pazienti privi di queste $[13,19]$ manifestazioni. Un inadeguato compenso ormonale, specialmente se protratto nel tempo, può avere notevoli implicazioni cliniche, come età ossea avanzata e bassa statura in pazienti pediatrici [20].

\section{Diagnosi}

L'esame obiettivo, pur rappresentando il primo step diagnostico nell'individuazione dei TARTs, può spesso trarre in inganno: infatti, tali lesioni sono di dimensioni variabili e sono rilevabili alla palpazione solo se presentano un diametro superiore ai due centimetri, a causa della loro localizzazione mediastinica.

Per questo motivo, la diagnosi dei tumori testicolari di origine surrenalica è prettamente strumentale: ad oggi, il gold-standard è rappresentato dall'ecografia testicolare. All'ecografia i TARTs appaiono come lesioni solide, tipicamente ipoecogene, con margini ben definiti, localizzate all'interno del mediastino testicolare, bilateralmente, caratterizzate da marcata vascolarizzazione interna allo studio Color-Doppler. Nelle lesioni di dimensioni maggiori si può osservare la presenza di zolle iperecogene intralesionali (Fig. 1) [21].

La Risonanza Magnetica Nucleare (RMN) del testicolo assume invece un ruolo diagnostico di secondo livello rispetto all'ecografia, che non solo è maggiormente economica e accessibile, ma può vantare una sensibilità sostanzialmente sovrapponibile alla RMN: entrambe le metodiche permettono, infatti, di rilevare anche lesioni di pochi millimetri di diametro.

Alla RMN i TARTs appaiono nettamente definiti rispetto al parenchima testicolare, iso- o iper-intensi in immagini T1-pesate, ipointensi in immagini T2-pesate, con omogeneo potenziamento post-contrastografico [21].
L'esame istologico tramite biopsia eco-guidata delle lesioni non è raccomandato, specialmente in presenza di criteri ultrasonografici e clinici già suggestivi di TARTs. Talvolta, però, può essere necessario al fine di eseguire una corretta diagnosi differenziale, poiché molte delle caratteristiche morfologiche dei TARTs, soprattutto se di piccole dimensioni, possono essere riscontrate in un'altra tipologia di lesioni testicolari, quali i tumori a cellule del Leydig (LCT) [22]. Entrambi, infatti, si presentano all'ecografia come lesioni solide, ipoecogene e ipervascolarizzate, distinguendosi essenzialmente per la bilateralità e per la localizzazione: i TARTs, tipicamente bilaterali, si localizzano in corrispondenza della rete testis, mentre gli LCTs, generalmente unilaterali, possono presentare una localizzazione variabile (Fig. 2) [23].

In casi particolarmente dubbi la biopsia risulta dirimente: sebbene entrambe le lesioni siano accomunate dalla presenza di grandi cellule poligonali con abbondante citoplasma eosinofilo e nuclei rotondi, caratteristiche tipiche di cellule steroido-secernenti [16], l'esame immunoistochimico evidenzia nei TARTs l'espressione proteica di marcatori di steroidogenesi surrenalica, assenti invece nei tumori a cellule del Leydig, che presentano nel $40 \%$ dei casi tipiche inclusioni citoplasmatiche, denominate "cristalli di Reinke" [24].

I TARTs di dimensioni maggiori entrano in diagnosi differenziale anche con i tumori a cellule germinali, in quanto entrambi ecograficamente possono apparire ipoecogeni, multifocali, a margini irregolari, con marcata vascolarizzazione intralesionale.

Una corretta diagnosi differenziale tra le diverse lesioni risulta quindi fondamentale, soprattutto alla luce della diversa gestione terapeutica: i tumori a cellule germinali necessitano di orchiectomia, i tumori a cellule di Leydig possono essere rimossi mediante testis sparing surgery $\mathrm{o}$, in casi selezionati, sottoposti a stretta surveillance clinica [25], mentre sarebbe opportuno asportare i TARTs solo in caso di severa sintomatologia algica o di fallimento della terapia medica [15].

\section{Gestione terapeutica}

Ad oggi, la gestione terapeutica dei TARTs è ancora oggetto di ampio dibattito scientifico, anche alla luce del fatto che non esistono chiare linee guida in merito [26].

Secondo le più recenti raccomandazioni, il trattamento di prima linea di queste lesioni consiste nell'intensificare il dosaggio dei glucocorticoidi al fine di sopprimere la secrezione ipofisaria di ACTH. Tale approccio sembra determinare maggiori effetti soprattutto negli stadi precoci di sviluppo dei TARTs, dimostrandosi efficace nel ridurre le dimensioni tumorali in alcuni dei pazienti trattati, ma non in tutti $[4,27]$. 
Fig. 1 Testicular adrenal rest tumors in un paziente di 21 anni affetto da CAH. All'ecografia i TARTs appaiono come lesioni solide, ipoecogene, con margini ben definiti, irregolari e sono tipicamente localizzate a livello del mediastino testicolare, bilateralmente e caratterizzate da marcata vascolarizzazione interna allo studio

Color-Doppler

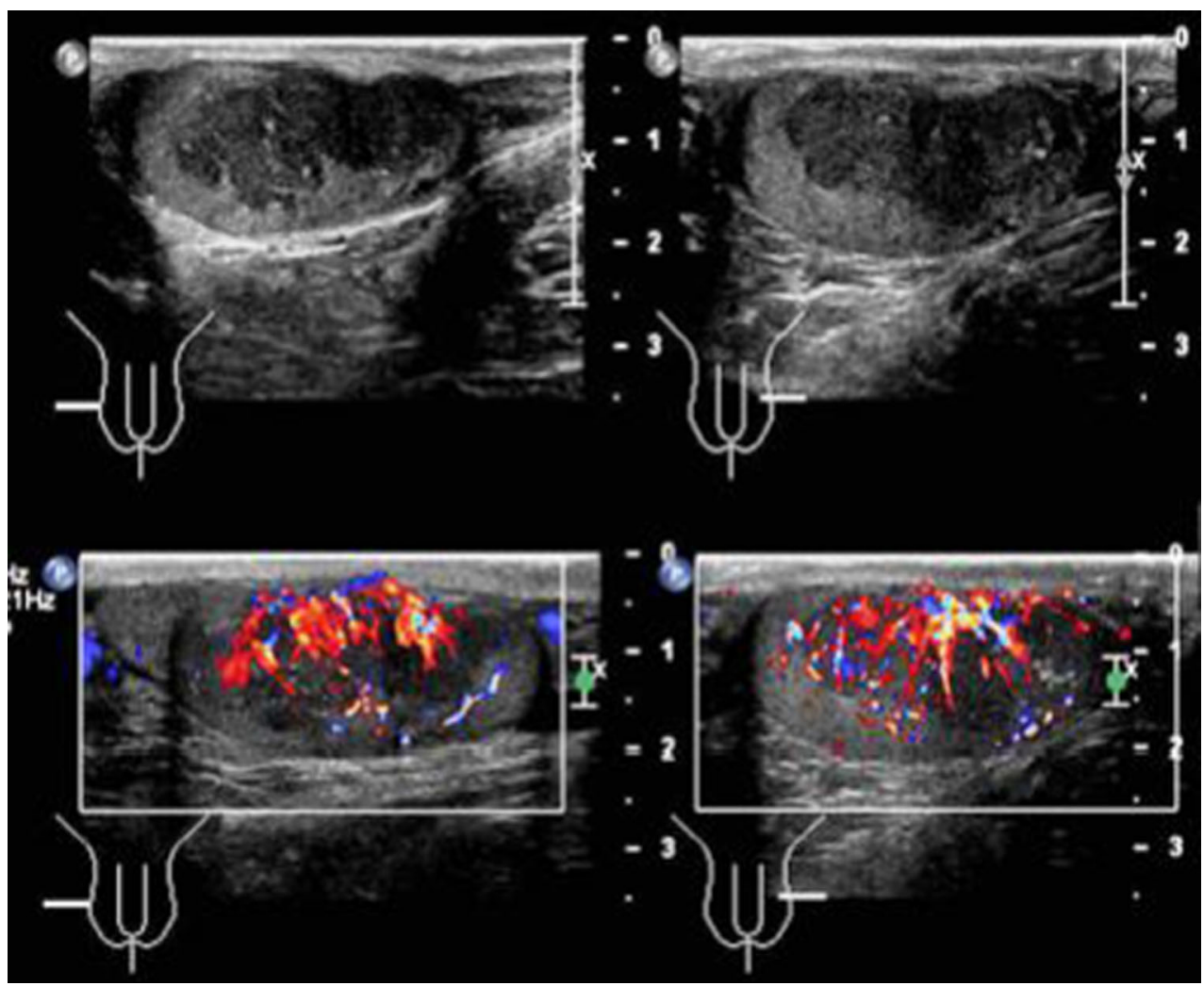

Fig. 2 Testicular adrenal rest tumors in un paziente di 27 anni affetto da CAH. I TARTs di piccole dimensioni (a) possono entrare in diagnosi differenziale con i tumori a cellule di Leydig (b), apparendo entrambi all'ecografica come piccole lesioni solide, ipoecogene, a margini ben definiti. A differenza dei tumori a cellule di Leydig, i TARTs sono tipicamente bilaterali e hanno origine a livello del mediastino testicolare

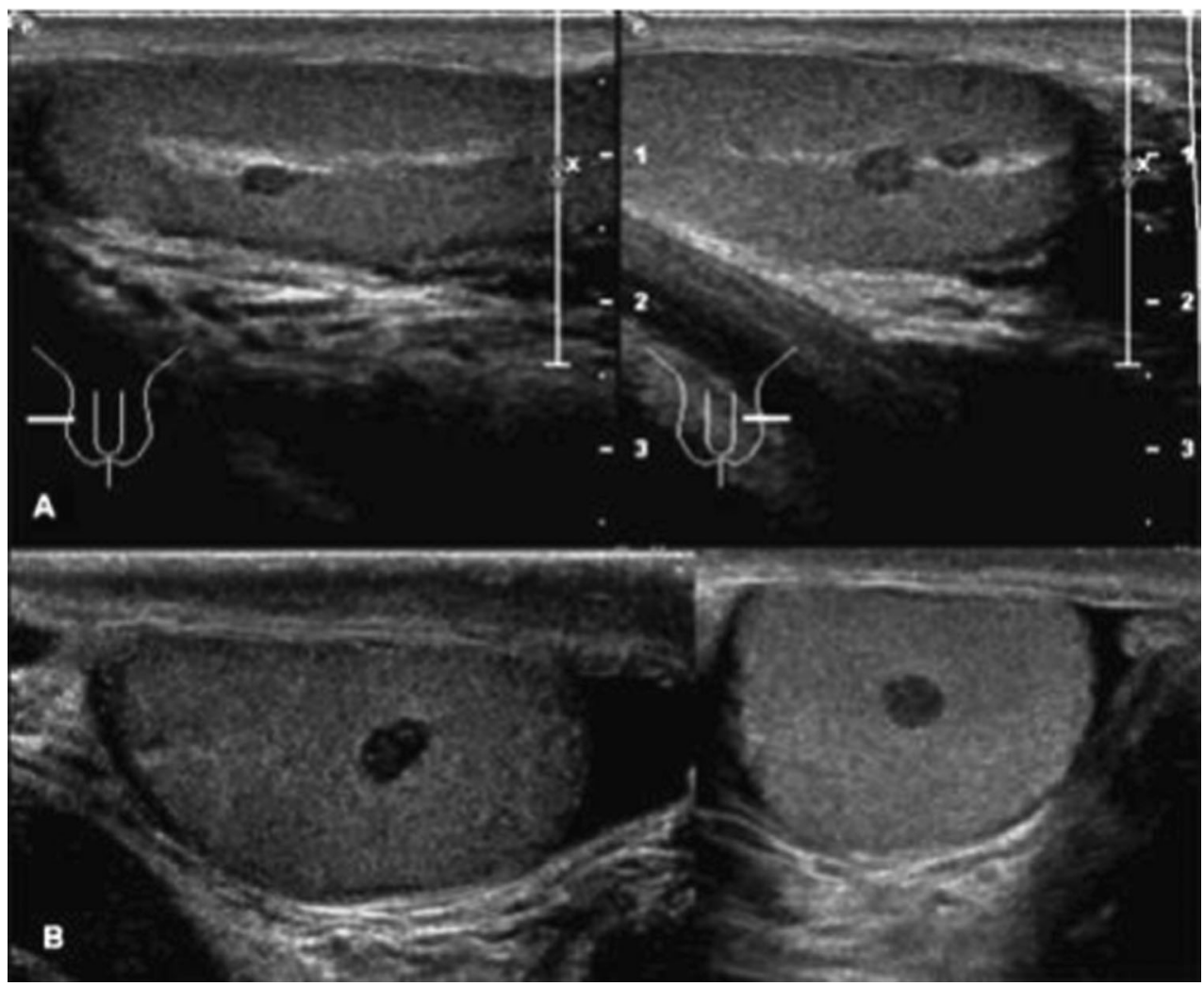

Inoltre, non è chiaro se il contributo della terapia corticosteroidea alla riduzione dei TARTs sia dovuto all'incremento della dose totale di glucocorticoidi, alla somministrazione anti-circadiana degli stessi o all'utilizzo di formulazioni a 
base di steroidi a più lunga emivita (desametasone piuttosto che prednisone). Ad ogni modo, l'adozione di un dosaggio modificato di glucocorticoidi non sembra determinare un significativo miglioramento dei parametri seminologici [18] e, allo stesso tempo, aumenta il rischio di sviluppo di effetti collaterali legati all'eccesso di glucocorticoidi, come ipertensione arteriosa, striae rubrae, incremento ponderale, riduzione dell' altezza target negli adolescenti.

Eventuali vantaggi di un regime terapeutico a base di formulazioni long-acting di glucocorticoidi a dosaggi standard sono ancora oggetto di discussione, sebbene siano riportati in letteratura recenti casi di regressione tumorale e di ripristino della fertilità, evidenziando ulteriormente la necessità di un approccio terapeutico individualizzato per ciascun paziente [28].

Il trattamento chirurgico via testis-sparing surgery è stato utilizzato su piccole coorti di pazienti con TARTs affetti da $\mathrm{CAH}$; tale trattamento non determina un significativo miglioramento della funzione gonadica nel postoperatorio [15], né esclude, peraltro, un'eventuale ricomparsa dei TARTs, con ulteriore danno testicolare. La chirurgia risulta, pertanto, indicata unicamente in caso di fallimento della terapia medica o di severa sintomatologia dolorosa a livello testicolare.

Va inoltre sottolineato che il trattamento delle forme classiche di $\mathrm{CAH}$ in maschi adolescenti, la categoria maggiormente interessata dai TARTs, risulta generalmente subottimale a causa di una compliance terapeutica spesso inadeguata. In questa classe di pazienti, spesso adeguatamente virilizzata e completamente asintomatica, un'aderenza soddisfacente al regime terapeutico rappresenta l'eccezione, piuttosto che la norma. Appare quindi fondamentale un attento counseling andrologico, al fine di educarli circa il rischio di sviluppo dei TARTs e il loro potenziale impatto sulla fertilità, offrendo altresì la possibilità di effettuare crioconservazione del liquido seminale [29]. Le linee guida raccomandano, nei maschi affetti da $\mathrm{CAH}$, uno screening ecografico precoce e periodico, con ecografia annuale a partire dagli otto anni e biennale a partire dal raggiungimento dell'età adulta [26].

In sintesi, ad oggi non esiste una terapia specifica in grado di trattare o impedire la formazione dei TARTs. Future prospettive di ricerca dovrebbero concentrarsi sull'individuazione di nuovi target terapeutici in grado di prevenirne la formazione: una concreta possibilità potrebbe essere rappresentata da farmaci inibitori dell' ACTH, non ancora disponibili in commercio, tenendo presente che tale ormone sembra essere il principale fattore coinvolto nel loro sviluppo.

Un recente studio ha analizzato gli effetti della somministrazione di un antagonista del recettore del CRH in donne con $\mathrm{CAH}$, riportando un decremento dose-dipendente dei livelli di ACTH e 17-OH progesterone. Questo farmaco, finora mai sperimentato in maschi con $\mathrm{CAH}$ affetti da adrenal rest tumors, potrebbe dunque rappresentare un interessante oggetto di ricerca al fine di prevenire la formazione dei TARTs [30].

\section{Conclusioni}

I TARTs rappresentano una complicanza rilevante nei pazienti maschi affetti da $\mathrm{CAH}$, più frequente nelle forme severe di malattia e con prevalente insorgenza durante la pubertà. Elevati livelli di ACTH sembrano essere il principale fattore patogenetico coinvolto nello sviluppo e nella progressione dei TARTs, ma non l'unico, essendo coinvolti anche LH e ATII. Attualmente le linee guida raccomandano di effettuare uno screening ecografico precoce nei maschi affetti da $\mathrm{CAH}$, sin dall'infanzia, di adeguare il dosaggio di glucocorticoidi al fine di normalizzare i livelli di ACTH e di offrire un counseling personalizzato riguardo la prospettiva di fertilità e la possibilità di effettuare una crioconservazione. L'individuazione precoce dei TARTs e un attento follow-up ecografico risultano, pertanto, fondamentali nel garantire il mantenimento della funzione testicolare.

Funding Note Open Access funding provided by Università degli Studi di Roma La Sapienza within the CRUI-CARE Agreement.

Conflitto di interesse Gli autori Dario De Alcubierre, Riccardo Pofi e Carlotta Pozza dichiarano di non avere conflitti di interesse.

Consenso informato Lo studio presentato in questo articolo non ha richiesto sperimentazione umana.

Studi sugli animali Gli autori di questo articolo non hanno eseguito studi sugli animali.

Nota della casa editrice Springer Nature rimane neutrale in riguardo alle rivendicazioni giurisdizionali nelle mappe pubblicate e nelle affiliazioni istituzionali.

Open Access This article is licensed under a Creative Commons Attribution 4.0 International License, which permits use, sharing, adaptation, distribution and reproduction in any medium or format, as long as you give appropriate credit to the original author(s) and the source, provide a link to the Creative Commons licence, and indicate if changes were made. The images or other third party material in this article are included in the article's Creative Commons licence, unless indicated otherwise in a credit line to the material. If material is not included in the article's Creative Commons licence and your intended use is not permitted by statutory regulation or exceeds the permitted use, you will need to obtain permission directly from the copyright holder. To view a copy of this licence, visit http://creativecommons.org/licenses/by/4.0/.

\section{Bibliografia}

1. Engels M, Span PN, van Herwaarden AE et al (2019) Testicular adrenal rest tumors: current insights on prevalence, characteristics, origin, and treatment. Endocr Rev 40:973-987 
2. Claahsen-van der Grinten HL, Sweep FC, Blickman JG et al (2007) Prevalence of testicular adrenal rest tumours in male children with congenital adrenal hyperplasia due to 21-hydroxylase deficiency. Eur J Endocrinol 157:339-344

3. Falhammar H, Nystrom HF, Ekstrom U et al (2012) Fertility, sexuality and testicular adrenal rest tumors in adult males with congenital adrenal hyperplasia. Eur J Endocrinol 166:441-449

4. Ashley RA, McGee SM, Isotaolo PA et al (2007) Clinical and pathological features associated with the testicular tumor of the adrenogenital syndrome. J Urol 177:546-549

5. Krieger DT, Samojlik E, Bardin CW (1978) Cortisol and androgen secretion in a case of Nelson's syndrome with paratesticular tumors: response to cyproheptadine therapy. J Clin Endocrinol Metab 47:837-844

6. Jin HY, Choi JH, Kim GH et al (2011) Testicular adrenal rest tumors in a patient with untreated congenital adrenal hyperplasia. Korean J Pediatr 54:137-140

7. Reisch N, Rottenkolber M, Greifenstein A et al (2013) Testicular adrenal rest tumors develop independently of long-term disease control: a longitudinal analysis of 50 adult men with congenital adrenal hyperplasia due to classic 21-hydroxylase deficiency. J Clin Endocrinol Metab 98:E1820-E1826

8. Smeets EE, Span PN, van Herwaarden AE et al (2015) Molecular characterization of testicular adrenal rest tumors in congenital adrenal hyperplasia: lesions with both adrenocortical and Leydig cell features. J Clin Endocrinol Metab 100:E524-E530

9. Rutgers JL, Young RH, Scully RE (1988) The testicular "tumor" of the adrenogenital syndrome. a report of six cases and review of the literature on testicular masses in patients with adrenocortical disorders. Am J Surg Pathol 12:503-513

10. Reisch N, Flade L, Scherr M et al (2009) High prevalence of reduced fecundity in men with congenital adrenal hyperplasia. J Clin Endocrinol Metab 94:1665-1670

11. Bouvattier C, Esterle L, Renoult-Pierre P et al (2015) Clinical outcome, hormonal status, gonadotrope axis, and testicular function in 219 adult men born with classic 21-hydroxylase deficiency. A French national survey. J Clin Endocrinol Metab 100:2303-2313

12. Falhammar H, Frisen L, Norrby $C$ et al (2017) Reduced frequency of biological and increased frequency of adopted children in males with 21-hydroxylase deficiency: a Swedish population-based national cohort study. J Clin Endocrinol Metab 102:4191-4199

13. Engels M, Gehrmann K, Falhammar H et al (2018) Gonadal function in adult male patients with congenital adrenal hyperplasia. Eur J Endocrinol 178:285-294

14. Claahsen-van der Grinten HL, Otten BJ, Hermus AR et al (2008) Testicular adrenal rest tumors in patients with congenital adrenal hyperplasia can cause severe testicular damage. Fertil Steril 89:597-601

15. Claahsen-van der Grinten HL, Otten BJ, Takahashi S et al (2007) Testicular adrenal rest tumors in adult males with congenital adrenal hyperplasia: evaluation of pituitary-gonadal function before and after successful testis-sparing surgery in eight patients. J Clin Endocrinol Metab 92:612-615
16. Claahsen-van der Grinten HL, Otten BJ, Sweep FC et al (2007) Testicular tumors in patients with congenital adrenal hyperplasia due to 21-hydroxylase deficiency show functional features of adrenocortical tissue. J Clin Endocrinol Metab 92:3674-3680

17. Turcu AF, Rege J, Chomic R et al (2015) Profiles of 21-carbon steroids in 21-hydroxylase deficiency. J Clin Endocrinol Metab 100:2283-2290

18. Mazzilli R, Stigliano A, Delfino M et al (2019) The high prevalence of testicular adrenal rest tumors in adult men with congenital adrenal hyperplasia is correlated with ACTH levels. Front Endocrinol (Lausanne) 10:335

19. Nermoen I, Rorvik J, Holmedal SH et al (2011) High frequency of adrenal myelolipomas and testicular adrenal rest tumours in adult Norwegian patients with classical congenital adrenal hyperplasia because of 21-hydroxylase deficiency. Clin Endocrinol (Oxf) 75:753-759

20. Kim MS, Goodarzian F, Keenan MF et al (2017) Testicular adrenal rest tumors in boys and young adults with congenital adrenal hyperplasia. J Urol 197:931-936

21. Stikkelbroeck NM, Suliman HM, Otten BJ et al (2003) Testicular adrenal rest tumours in postpubertal males with congenital adrenal hyperplasia: sonographic and MR features. Eur Radiol 13:15971603

22. Ali HH, Samkari A, Arabi H (2013) Testicular adrenal rest "tumor" or Leydig cell tumor? A report of a challenging case with literature review. Avicenna J Med 3:15-19

23. Rich MA, Keating MA (2000) Leydig cell tumors and tumors associated with congenital adrenal hyperplasia. Urol Clin North Am 27:519-528

24. Kim I, Young RH, Scully RE (1985) Leydig cell tumors of the testis. A clinicopathological analysis of 40 cases and review of the literature. Am J Surg Pathol 9:177-192

25. Pozza C, Pofi R, Tenuta M et al (2019) Clinical presentation, management and follow-up of 83 patients with Leydig cell tumors of the testis: a prospective case-cohort study. Hum Reprod 34:1389-1403

26. Speiser PW, Arlt W, Auchus RJ et al (2018) Congenital adrenal hyperplasia due to steroid 21-hydroxylase deficiency: an Endocrine Society clinical practice guideline. J Clin Endocrinol Metab 103:4043-4088

27. Tanaka M, Enatsu N, Chiba K, Fujisawa M (2018) Two cases of reversible male infertility due to congenital adrenal hyperplasia combined with testicular adrenal rest tumor. Reprod Med Biol 17:93-97

28. Jha S, El-Maouche D, Marko J et al (2019) Individualizing management of infertility in classic congenital adrenal hyperplasia and testicular adrenal rest tumors. J Endocr Soc 3:2290-2294

29. Merke DP, Poppas DP (2013) Management of adolescents with congenital adrenal hyperplasia. Lancet Diabetes Endocrinol $1: 341-352$

30. Turcu AF, Spencer-Segal JL, Farber RH et al (2016) Single-dose study of a corticotropin-releasing factor receptor-1 antagonist in women with 21-hydroxylase deficiency. J Clin Endocrinol Metab 101:1174-1180 